\title{
CARACTERÍSTICAS MICROBIOLÓGICAS Y TERAPÉUTICAS DE LA SEPSIS NEONATAL CONFIRMADA EN UN HOSPITAL DE LIMA, PERÚ
}

\author{
Giancarlo Alvarado-Gamarra ${ }^{1,2, a}$, Katherine M. Alcalá-Marcos ${ }^{1,2, a}$, Diego M. Abarca-Alfaro $3, a$, Victoria Bao-Castro $4, b$
}

\begin{abstract}
RESUMEN
Objetivos. Describir las características microbiológicas y terapéuticas de la sepsis neonatal confirmada. Además, determinar su incidencia y mortalidad en el Hospital Nacional Arzobispo Loayza (HNAL) del 2011 al 2012, Lima, Perú. Materiales y métodos. Estudio longitudinal retrospectivo. Se realizó seguimiento a neonatos con sepsis probable, evaluando aparición de hemocultivo positivo. Las características de los neonatos fueron obtenidas de las historias clínicas. Se determinó la incidencia acumulada de sepsis confirmada, tasa de mortalidad y tasa de letalidad. Para las variables cuantitativas se usó la media con desviación estándar o la mediana con rangos intercuartílicos, según corresponda. Para variables cualitativas se usó frecuencias relativas y porcentajes. Resultados. La incidencia de sepsis confirmada fue de 4,1 por cada 1000 nacidos vivos (IC 95\%: 2,7-5,5). La tasa de mortalidad fue de 0,97 por cada 1000 nacidos vivos (IC 95\%: 0,29-1,63) y la tasa de letalidad fue de 23,5\% (IC 95\%: 9-37). Los aislamientos más frecuentes fueron de Staphylococcus coagulasa negativo, seguido por el Staphylococcus aureus; ambos con altas tasas de resistencia a oxacilina (90 y $66,6 \%$, respectivamente). A un $67 \%$ se inició amipicilina/amikacina o ampicilina/cefotaxima. Se rotó de antibióticos a un $58 \%$ de neonatos por mala evolución clínica/laboratorial o por resultado de hemocultivo, iniciando vancomicina a la mayoría $(42,8 \%)$. Conclusiones. Existe una elevada incidencia de sepsis confirmada y una elevada tasa de letalidad. La terapéutica administrada no estuvo acorde con el perfil microbiológico.
\end{abstract}

Palabras clave: Sepsis; Recién nacido; Tasa de mortalidad; Incidencia (fuente: DeCS BIREME).

\section{MICROBIOLOGICAL AND THERAPEUTIC CHARACTERISTICS OF CONFIRMED NEONATAL SEPSIS AT A HOSPITAL IN LIMA, PERU}

\begin{abstract}
Objectives. To describe the microbiological and therapeutic characteristics of confirmed neonatal sepsis, and determine its incidence and mortality, at the National Hospital Arzobispo Loayza (HNAL) from 2011 to 2012, Lima, Peru. Materials and Methods. Retrospective longitudinal study. It was performed a monitoring to newborns with probable sepsis, and assessed the results from positive blood cultures. The newborns' characteristics were obtained from medical records. The acumulated incidences of confirmed sepsis, mortality and fatality rates were determined. Quantitative variables were reported as men \pm standard deviation or median (interquartile range). Qualitative variables were reported as relative frequencies and percentages. Results. The incidence of confirmed sepsis was 4.1 per 1000 live births (95\% confidence interval [Cl]: 2.7-5.5). The mortality rate was 0.97 per 1000 live births (95\% Cl: $0.29-1.63)$ and the fatality rate was $23.5 \%(95 \% \mathrm{Cl}: 9-37)$. The most commonly isolated organism was coagulase-negative Staphylococcus, followed by Staphylococcus aureus, both with high rates of oxacillin resistance ( $90 \%$ and $66.6 \%$, respectively). A $67 \%$ of the newborns were treated using ampicillin/amikacin or ampicillin/cefotaxime. Antibiotics were changed in $58 \%$ of the newborns because of poor clinical/laboratory evolution or blood culture results. In most cases the seconds antibiotic was vancomycin $(42.8 \%)$. Conclusions. There is a high incidence of confirmed sepsis and a high fatality rate. The administered treatments were not consistent with the microbiological profiles.
\end{abstract}

Key words: Sepsis; Infant, newborn; Mortality rate; Incidence (source: MeSH NLM).

\footnotetext{
Sociedad Científica de Estudiantes de Medicina Villarrealinos (SOCEMVI). Universidad Nacional Federico Villarreal. Lima, Perú.

Facultad de Medicina "Hipólito Unanue". Universidad Nacional Federico Villarreal. Lima, Perú.

Facultad de Medicina. Universidad Ricardo Palma. Lima, Perú.

Hospital Nacional Arzobispo Loayza. Lima, Perú.

Médico cirujano; ${ }^{\mathrm{b}}$ pediatra neonatólogo

Este artículo forma parte de la tesis: Alvarado-Gamarra AG. Incidencia, mortalidad, perfil microbiológico-terapéutico y factores asociados a hemocultivos positivos en neonatos con sepsis probable de un hospital del Perú, 2011-2012 [Tesis para optar el título profesional de médico cirujano]. Lima: Facultad de Medicina "Hipólito Unanue", Universidad Nacional Federico Villarreal; 2014.

Recibido: 30-06-15 Aprobado: 09-03-16
}

Citar como: Alvarado-Gamarra G, Alcalá-Marcos KM, Abarca-Alfaro DM, Bao-Castro V. Características microbiológicas y terapéuticas de la sepsis neonatal confirmada en un hospital de Lima, Perú. Rev Peru Med Exp Salud Publica. 2016;33(1):74-82. doi: 10.17843/rpmesp.2016.331.2010 


\section{INTRODUCCIÓN}

La reducción de la mortalidad infantil es uno de los objetivos de desarrollo del milenio, la mortalidad neonatal contribuye con más del $40 \%{ }^{(1)}$. La mortalidad neonatal en el Perú ha disminuido en los tres últimos quinquenios, en el 2013 se han reportado 12 defunciones por 1000 nacidos vivos ${ }^{(2)}$; sin embargo, sigue siendo elevada en comparación con otros países de la región. En Perú, las principales causas de mortalidad neonatal son la prematuridad y las infecciones ${ }^{(3)}$, en los recién nacidos de bajo peso (1500 a $2500 \mathrm{~g})$ la primera causa son las infecciones ${ }^{(4)}$. En un estudio realizado en Ucayali y Huánuco, se ha reportado que la sepsis neonatal es la principal causa básica de mortalidad neonatal (1). De este modo, el estudio de la sepsis neonatal resulta relevante para disminuir la mortalidad neonatal e infantil en el Perú.

En la sepsis neonatal los principales aspectos a considerar son el diagnóstico precoz y la administración de antibióticos. Para evitar la demora en el diagnóstico es necesario un elevado grado de sospecha, basado en manifestaciones clínicas, exámenes auxiliares y factores de riesgo compatibles con sepsis neonatal ${ }^{(5)}$. Esto resulta importante al redundar en un menor tiempo de inicio de tratamiento $\mathrm{y}$, probablemente, en disminuir la elevada carga de morbimortalidad asociada a sepsis neonatal ${ }^{(6)}$. En Estados Unidos, del 2005 al 2008, la mortalidad asociada a sepsis neonatal fue de $10,9 \%{ }^{(7)}$. En Perú, no se ha reportado la mortalidad asociada. Se presume que debe ser mayor. Además, no se ha reportado si el tratamiento empírico instaurado está acorde con el perfil microbiológico de cada hospital, resultando una probable práctica habitual la aplicación de las recomendaciones terapéuticas de guías de otros países.

Es necesario evaluar el perfil microbiológico para que sirva de pauta a la terapia empírica inicial, este perfil es variable según país, ciudad u hospital. En un estudio en Lima, los gérmenes aislados con más frecuecia fueron Staphylococcus epidermidis $(38,3 \%$ ) y el Staphylococcus aureus (12\%). También existen otros reportes nacionales en los que predominan los gérmenes Gram positivos, siendo el más frecuente el S. epidermidis ${ }^{(8)}$.En los Estados Unidos predominan las cepas de Streptococcus del grupo B (GBS) y los Gram negativos ${ }^{(9,10)}$.En Latinoamérica, los GBS son poco frecuentes, pero los Gram negativos son prevalentes ${ }^{(11,12)}$. Actualmente, en países desarrollados y en vías de desarrollo existe tendencia al aislamiento de Staphylococcus spp., en Estados Unidos se ha descrito esto en neonatos sometidos a procedimientos invasivos y en sepsis tardía ${ }^{(7)}$. Evidenciar el perfil microbiológico local resulta necesario para un adecuado manejo de la sepsis neonatal.
Por lo tanto, con el fin de conocer la morbimortalidad asociada a sepsis neonatal, el objetivo de este estudio fue describir las características microbiológicas y terapéuticas de la sepsis neonatal confirmada. Además, determinar su incidencia y mortalidad en el Hospital Nacional Arzobispo Loayza (HNAL) en Lima, Perú.

\section{MATERIALES Y MÉTODOS}

\section{DISEÑO Y POBLACIÓN}

Estudio longitudinal retrospectivo. Se realizó el seguimiento a neonatos con sepsis probable, atendidos en la unidad de cuidados intensivos e intermedios del servicio de neonatología del HNAL de Lima, Perú en el 2011 y 2012.

\section{RECOLECCIÓN DE DATOS}

Se identificaron los casos de sepsis probable empleando la base de datos del servicio de neonatología. Luego, se realizó el seguimiento durante la hospitalización evaluando la aparición de hemocultivo positivo, fue suficiente un hemocultivo positivo para confirmar la sepsis probable. No se ha considerado hemocultivos positivos de forma aislada. Se excluyó a los neonatos con cultivo positivo a hongos. No hubo casos de meningitis confirmada. No se estudió la colonización para GBS en las gestantes. Se obtuvieron 34 casos de sepsis confirmada. De estos, ocho neonatos fallecieron. No se obtuvo las historias clínicas de estos ocho, algunas no se encontraban en sus lugares de origen, otras fueron enviadas a otras instancias sin regreso. Para la descripción de las características de los neonatos no se incluyó a estos ocho. Se identificó que fueron casos confirmados por estar consignados en la base de datos del servicio de neonatología. Se utilizó una ficha de recolección para consignar las variables del estudio al revisar las historias clínicas de los neonatos con sepsis confirmada.

\section{VARIABLES DE ESTUDIO}

Se definió a los neonatos con sepsis probable en base a manifestaciones clínicas, factores de riesgo maternos/ neonatales, y exámenes auxiliares alterados, acorde con la definición operacional propuesta por el subsistema nacional de vigilancia epidemiológica perinatal y neonatal del Ministerio de Salud del Perú (5). De estos, aquellos con hemocultivo positivo fueron considerados como sepsis confirmada. Se describió las características microbiológicas (aislamiento bacteriano, tasa de resistencia a oxacilina y vancomicina, expresión de resistencia compatible con betalactamasas de espectro extendido, BLEE) y las características terapéuticas (antibióticos administrados, tiempo de administración, rotación de antibióticos). Se definió sepsis precoz a la diagnosticada dentro de las primeras $72 \mathrm{~h}$, y tardía luego 
de este periodo. Además, se describió las características maternas, clínicas, de laboratorio, invasivas y comorbilidades de los neonatos con sepsis confirmada.

\section{CONSIDERACIONES ÉTICAS}

El estudio fue revisado y aprobado por el Comité de Investigación de la Facultad de Medicina "Hipólito Unanue" de la Universidad Nacional Federico Villarreal, y por la Oficina de Docencia e Investigación del HNAL. Se respetó la confidencialidad de los datos obtenidos.

\section{ANÁLISIS ESTADÍSTICO}

Los datos obtenidos ingresaron a una base de datos de Excel v.2010; posteriormente, se analizaron con el paquete estadístico SPSS versión 19. Se determinó la incidencia acumulada de sepsis neonatal probable y confirmada. También se determinó la tasa de mortalidad (neonatos con sepsis confirmada muertos sobre los neonatos atendidos) y la tasa de letalidad (neonatos con sepsis confirmada muertos sobre los neonatos con sepsis confirmada). Para las variables cuantitativas se usó la media con desviación estándar o la mediana con sus rangos intercuartílicos acorde a las pruebas de normalidad (test de Kolmogorov-Smirnov). Para variables cualitativas se usó frecuencias relativas y porcentajes.

\section{RESULTADOS}

\section{CARACTERÍSTICAS DE LA COHORTE ESTUDIADA}

La cantidad de neonatos atendidos fueron 8261, en el año 2011 se atendió a 4170 y en el 2012 a 4091 . El estudio incluyó a 200 neonatos con diagnóstico de sepsis probable para iniciar el seguimiento. El $55,5 \%$ fueron hombres y un $88,5 \%$ nació en el hospital. La mediana del peso al nacer fue $1855 \mathrm{~g}$. La mediana de la edad gestacional fue 34,5 semanas y la mayoría fueron prematuros. Respecto a las características maternas, la mayoría procedía de Lima. La mediana de la edad de las madres fue de 26,5 años, la mayoría era conviviente y había concluido la secundaria. La mayoría tuvo un inadecuado control prenatal. A un $67 \%$ se le realizó cesárea (Tabla 1).

\section{INCIDENCIA Y MORTALIDAD DE SEPSIS NEONATAL CONFIRMADA}

El seguimiento se realizó durante la hospitalización (mediana de 30 días, con un rango de 15,5 a 55,5 días) o hasta la muerte de los neonatos. El diagnóstico de sepsis probable se dio en 60 horas (mediana), rango de 0 a 384 horas. La confirmación del diagnóstico fue a
Tabla 1. Características de la cohorte de estudio $(\mathrm{N}=200)$. Hospital Nacional Arzobispo Loayza, 20112012. Lima, Perú

\begin{tabular}{|c|c|}
\hline Características & $\%(n / N)$ \\
\hline \multicolumn{2}{|l|}{ NEONATALES } \\
\hline \multicolumn{2}{|l|}{ Sexo } \\
\hline Masculino & $55,5(111 / 200)$ \\
\hline Femenino & $44,5(89 / 200)$ \\
\hline \multicolumn{2}{|l|}{ Nacido en el hospital } \\
\hline Sí & $88,5(177 / 200)$ \\
\hline No & $11,5(23 / 200)$ \\
\hline Peso al nacer & $1855 g(1280-3002)^{*}$ \\
\hline \multicolumn{2}{|l|}{$\begin{array}{l}\text { Clasificación del peso al } \\
\text { nacer }\end{array}$} \\
\hline Normal & $31(62 / 200)$ \\
\hline Bajo peso & $28,5(57 / 200)$ \\
\hline Muy bajo peso & $22,5(45 / 200)$ \\
\hline $\begin{array}{l}\text { Extremadamente bajo } \\
\text { peso }\end{array}$ & $13,5(27 / 200)$ \\
\hline Macrosómico & $4,5(9 / 200)$ \\
\hline Edad gestacional (EG) & 34,5 semanas $(31-38)^{*}$ \\
\hline \multicolumn{2}{|l|}{ Prematuro } \\
\hline Sí & $61(122 / 200)$ \\
\hline No & $39(78 / 200)$ \\
\hline \multicolumn{2}{|l|}{$\begin{array}{l}\text { Clasificación de } \\
\text { prematuros }\end{array}$} \\
\hline Tardío & $32,3(41 / 127)$ \\
\hline Moderado & $25,2(32 / 127)$ \\
\hline Extremo & $25,2(32 / 127)$ \\
\hline Muy extremo & $17,3(22 / 127)$ \\
\hline \multicolumn{2}{|l|}{ Peso según EG } \\
\hline AEG & $67,5(135 / 200)$ \\
\hline PEG & $26,5(53 / 200)$ \\
\hline GEG & $6(12 / 200)$ \\
\hline \multicolumn{2}{|l|}{ MATERNAS } \\
\hline Edad de la madre & 26,5 años $(21-31)^{*}$ \\
\hline \multicolumn{2}{|l|}{ Estado civil } \\
\hline Conviviente & $62(57 / 200)$ \\
\hline Soltera & $23(62 / 200)$ \\
\hline Casada & $14,5(27 / 200)$ \\
\hline Viuda & $0,5(9 / 200)$ \\
\hline \multicolumn{2}{|l|}{ Procedencia } \\
\hline Lima & $94,9(189 / 199)$ \\
\hline Provincia & $5,1(10 / 199)$ \\
\hline \multicolumn{2}{|l|}{ Nivel educativo } \\
\hline Analfabeta & $1,5(3 / 200)$ \\
\hline Primaria & $12,5(25 / 200)$ \\
\hline Secundaria & $66,5(133 / 200)$ \\
\hline Superior & $19,5(39 / 200)$ \\
\hline \multicolumn{2}{|l|}{ Controles prenatales } \\
\hline Inadecuado & $55,5(111 / 200)$ \\
\hline Adecuado & $44,5(89 / 200)$ \\
\hline \multicolumn{2}{|l|}{ Tipo de parto } \\
\hline Cesárea & $67(134 / 200)$ \\
\hline Vaginal & $33(66 / 200)$ \\
\hline $\begin{array}{l}\text { *mediana (rango intercuartílicc } \\
\text { EG: Edad Gestacional } \\
\text { AEG: Adecuado para la edac } \\
\text { PEG: Pequeño para la edad } \\
\text { GEG: Grande para la edad g }\end{array}$ & $\begin{array}{l}\text { cional } \\
\text { onal } \\
\text { hal }\end{array}$ \\
\hline
\end{tabular}


los 9 días (mediana) con una variación de 6 a 12,7 días. Se encontró 34 casos de sepsis confirmada, arrojando una incidencia acumulada de 4,1 por cada 1000 nacidos vivos (IC 95\%: 2,7-5,5). Ocho casos fallecieron durante la hospitalización. De los 26 casos restantes, 16 fueron sepsis confirmada precoz arrojando una incidencia acumulada de 1,93 por cada 1000 nacidos vivos (IC $95 \%$ : 1,0-2,8); y 10 fueron sepsis confirmada tardía, arrojando una incidencia acumulada de 1,21 por cada 1000 nacidos vivos (IC 95\%: 0,5-1,8). La incidencia de sepsis probable fue de 24,2 por cada 1000 nacidos vivos (IC 95\%: 21,0-27,4). En los neonatos con sepsis confirmada, la mediana del peso al nacer fue de $1525 \mathrm{~g}$ $(1076,2-3012,5)$, la mayoría $(70,5 \%, 24 / 34)$ tuvo un peso por debajo de lo normal al nacer, y un $64,7 \%$ (22/34) fueron prematuros, de estos, la mayoría fue prematuro extremo (36,3\%). Un 17\% (IC 95\%: 11,8-22,2) de neonatos con sepsis probable tuvieron hemocultivo positivo. La tasa de letalidad fue de un 23,5\% (8/34) (IC 95\%: 9-37), y la tasa de mortalidad fue de 0,97 por cada 1000 nacidos vivos (IC 95\%: 0,29-1,63). La tasa de letalidad aumentó a $75 \%(6 / 8)$ en neonatos con peso por debajo del percentil 25 , y a $62,5 \%$ (5/8) en prematuros.

\section{CARACTERISTTICAS MICROBIOLÓGICAS}

La mayoría de aislamientos fueron Gram positivos, el Staphylococcus coagulasa negativo (SGN) fue el germen más aislado, seguido por el Staphylococcus aureus. Klebsiella spp. fue la bacteria Gram negativa más aislada. Los SGN y Staphylococcus aureus fueron los agentes más frecuentes tanto en sepsis precoz como tardía. La tasa de resistencia a oxacilina en SGN fue un $90 \%$, en Staphylococcus aureus un $66,6 \%$ y la aparición de cepas BLEE en Gram negativos fue de $75 \%$. No se registró Staphylococcus aureus resistente a vancomicina (VRSA) ni Enterococcus resistente a vancomicina (VRE) (Tabla 2).

\section{CARACTERÍSTICAS TERAPÉUTICAS}

A todos los neonatos con sepsis probable se les administró antibióticos. La mediana del tiempo de administración fue de 14,5 días, resultando un $41,6 \%$ con administración prolongada. Se administró hasta tres combinaciones de antibióticos en un mismo neonato. En la primera combinación se usó principalmente amipicilina/amikacina $(41,6 \%)$ o ampicilina/cefotaxima (25\%), con una mediana del tiempo de administración de 7,5 días. De estos se rotó de antibiótico a un 58,3\%, a causa de una mala evolución clínica y alteraciones de exámenes auxiliares (50\%), y por el resultado del hemocultivo $(42,8 \%)$. En la segunda combinación los antibióticos más usados fueron vancomicina (42,8\%) o vancomicina/ceftazidima $(21,4 \%)$. La mediana del tiempo de administración fue 14 días. De estos a un $21,4 \%$

Tabla 2. Características microbiológicas de sepsis neonatal confirmada. Hospital Nacional Arzobispo Loayza, 2011-2012. Lima, Perú

\begin{tabular}{|c|c|c|c|}
\hline \multirow{2}{*}{ Microorganismos aislados* } & \multirow[b]{2}{*}{$\%(n / N)$} & \multicolumn{2}{|c|}{ Sepsis neonatal \% (n/N) } \\
\hline & & Precoz & Tardía \\
\hline Staphylococcus coagulasa negativo & $38,5(10 / 26)$ & $43,7(7 / 16)$ & $30(3 / 10)$ \\
\hline Staphylococcus aureus & $23,2(6 / 26)$ & $18,7(3 / 16)$ & $30(3 / 10)$ \\
\hline Klebsiella spp. & $15,4(4 / 26)$ & $12,5(2 / 16)$ & $20(2 / 10)$ \\
\hline Escherichia coli & $11,5(3 / 26)$ & $6,2(1 / 16)$ & $20(2 / 10)$ \\
\hline Pseudomonas aeruginosa & $3,8(1 / 26)$ & $6,2(1 / 16)$ & $0(0 / 10)$ \\
\hline Enterococcus spp. & $3,8(1 / 26)$ & $6,2(1 / 16)$ & $0(0 / 10)$ \\
\hline Estreptococo viridans & $3,8(1 / 26)$ & $6,2(1 / 16)$ & $0(0 / 10)$ \\
\hline \multicolumn{4}{|l|}{ Tipo Gram } \\
\hline Positivo & $69,2(18 / 26)$ & $75(12 / 16)$ & $60(6 / 10)$ \\
\hline Negativo & $30,8(8 / 26)$ & $25(4 / 16)$ & $40(4 / 10)$ \\
\hline $\begin{array}{l}\text { Staphylococcus coagulasa negativos resistente a } \\
\text { oxacilina }\end{array}$ & $90(9 / 10)$ & & \\
\hline Staphylococcus aureus resistente a meticilina (MRSA) & $66,6(4 / 6)$ & & \\
\hline Betalactamasas de espectro extendido (BLEE) & $75(6 / 8)$ & & \\
\hline
\end{tabular}

*No se obtuvo el tipo de microorganismo de los 34 casos de sepsis confirmada 
se decidió rotar de antibiótico, siendo los antibióticos usados meropenem o ciprofloxacino, administrándolos en un promedio de 9 días (Tabla 3).

Tabla 3. Características de los antibióticos administrados en la sepsis neonatal confirmada. Hospital Nacional Arzobispo Loayza, 2011-2012.Lima, Perú

\begin{tabular}{|c|c|}
\hline Características & $\%(n / N)$ \\
\hline $\begin{array}{l}\text { Tiempo de administración de } \\
\text { antibióticos }\end{array}$ & 14,5 días $(10-21)^{\star}$ \\
\hline Prolongada** & $41,6(10 / 24)$ \\
\hline No prolongada & $58,4(14 / 24)$ \\
\hline \multicolumn{2}{|l|}{ Antibióticos (primera combinación) } \\
\hline Amipicilina/Amikacina & $41,6(10 / 24)$ \\
\hline Ampicilina/Cefotaxima & $25(6 / 24)$ \\
\hline Meropenem & $8,3(2 / 24)$ \\
\hline Vancomicina/Ceftazidima & $8,3(2 / 24)$ \\
\hline Amikacina & $4,2(1 / 24)$ \\
\hline Ciprofloxacino & $4,2(1 / 24)$ \\
\hline Ampicilina/Cefotaxima/Metronidazol & $4,2(1 / 24)$ \\
\hline Vancomicina & $4,2(1 / 24)$ \\
\hline $\begin{array}{l}\text { Tiempo de administración } \\
\text { (primera combinación) }\end{array}$ & 7,5 días $(6-10)^{*}$ \\
\hline \multicolumn{2}{|l|}{ Motivo de rotación } \\
\hline Mala evolución clínica y de laboratorio & $50(7 / 14)$ \\
\hline Resultado del hemocultivo & $42,8(6 / 14)$ \\
\hline $\begin{array}{l}\text { Hemocultivo/mala evolución clínica y } \\
\text { de laboratorio }\end{array}$ & $7,2(1 / 14)$ \\
\hline \multicolumn{2}{|l|}{ Antibióticos (segunda combinación) } \\
\hline Vancomicina & $42,8(6 / 14)$ \\
\hline Vancomicina/Ceftazidima & $21,4(3 / 14)$ \\
\hline Meropenem & $14,5(2 / 14)$ \\
\hline Ampicilina/Cefotaxima & $7,1(1 / 14)$ \\
\hline Meropenem/Amikacina & $7,1(1 / 14)$ \\
\hline Vancomicina/Ciprofloxacino & $7,1(1 / 14)$ \\
\hline $\begin{array}{l}\text { Tiempo de administración } \\
\text { (segunda combinación) }\end{array}$ & 14 días $(9,5-15,25)^{*}$ \\
\hline \multicolumn{2}{|l|}{ Antibióticos (tercera combinación) } \\
\hline Meropenem & $66,7(2 / 3)$ \\
\hline Ciprofloxacino & $33,3(1 / 3)$ \\
\hline $\begin{array}{l}\text { Tiempo de administración } \\
\text { (tercera combinación) }\end{array}$ & 9 días $+/-1,73 \dagger$ \\
\hline
\end{tabular}

\section{OTRAS CARACTERÍSTICAS DE LOS NEONATOS CON SEPSIS CONFIRMADA}

Un $91,7 \%$ (22/24) presentaron alguna manifestación clínica. De estos, un $36,3 \%$ (8/22) presentó tres manifestaciones clínicas, y un $27,2 \%$ (6/22) presentó dos. La mayoría presentó síndrome de distrés respiratorio, seguido de ictericia neonatal y apnea, estos predominaron tanto en sepsis precoz y tardía, agregándose temperatura $>38{ }^{\circ} \mathrm{C}$, bradicardia fetal, y cianosis central en la sepsis tardía. En relación a los exámenes auxiliares, un $96 \%(23 / 24)$ presentó alguna alteración. Un 34,8\% (8/23) presentó al menos dos exámenes auxiliares alterados y un $30,4 \%(7 / 23)$ presentó tres. La mayoría presentó trombocitopenia e hipoglicemia. Los procedimientos invasivos y situaciones que predispusieron su uso fueron frecuentes. Las enfermedades que se presentaron fueron las relacionadas con prematuros (Tabla 4). Con respecto a las características maternas, a un $67,6 \%(23 / 34)$ se realizó cesárea. Un 29,4\% (10/34) tuvo antecedente de RPM, con una mediana de 19 horas (rango de 6-39,7 horas). Un $14,7 \%(5 / 34)$ cursó con RPM $>18$ h y un $20,6 \%(7 / 34)$ con RPM>6 h. Un 12,5\% (3/24) presentó líquido meconial denso al nacer.

\section{DISCUSIÓN}

La sepsis es una causa relevante de morbilidad y mortalidad en neonatos. En el Perú, se ha reportado un descenso en la tasa de mortalidad neonatal en los últimos años ${ }^{(2)}$; este descenso se evidencia en varios hospitales, en los que la mortalidad neonatal precoz (hasta los 7 días de nacido) disminuye de 10,45 a 9,03 por cada 1000 nacidos vivos desde el 2000 al $20088^{(13,14)}$. A pesar de lo descrito, la tasa de mortalidad neonatal aún es elevada en comparación con países desarrollados, y el estudio de la sepsis neonatal podría contribuir en disminuirla aún más.

La incidencia de sepsis confirmada hallada en el HNAL (de 4,1 por 1000 nacidos vivos) fue elevada en comparación con la reportada en los Estados Unidos (0,77 a 1 por 1000 nacidos vivos) ${ }^{(9,15,16)}$ y el MINSA (2,5 por 1000 nacidos vivos) (17). Además, en el HNAL los casos de sepsis confirmada han aumentado en los últimos años, en el 2005 se reportó una incidencia de 1,74 por cada 1000 nacidos vivos (datos no publicados). En América del Sur y el Caribe la incidencia es de 3,59 a 8,91 por cada 1000 nacidos vivos ${ }^{(18)}$. En Chile, algunos centros han reportado un caso por cada 1000 nacidos vivos ${ }^{(19)}$, similar a los países desarrollados. En México, algunos centros han reportado hasta 160,93 casos por cada 1000 nacidos vivos ${ }^{(11)}$. En el Perú también existe variabilidad, probablemente por la heterogeneidad en la definición de sepsis, por los métodos microbiológicos usados y por la recepción de neonatos 
Tabla 4. Características clínicas, de laboratorio, invasivas y comorbilidades de neonatos con sepsis confirmada. Hospital Nacional Arzobispo Loayza, 2011-2012. Lima, Perú

\begin{tabular}{|c|c|c|c|}
\hline \multirow{2}{*}{$\begin{array}{l}\text { Características } \\
\text { Manifestaciones clínicas }\end{array}$} & \multirow[t]{2}{*}{$\%(n / N)$} & \multicolumn{2}{|c|}{ Sepsis neonatal \% $(n / N)$} \\
\hline & & Precoz & Tardía \\
\hline Síndrome de distrés respiratorio & $24,6(14 / 57)$ & $57,1(8 / 14)$ & $42,8(6 / 14)$ \\
\hline Ictericia neonatal indirecta $90(9 / 10)$, directa $10(1 / 10)$ & $17,5(10 / 57)$ & $80(8 / 10)$ & $20(2 / 10)$ \\
\hline Apnea & $15,8(9 / 57)$ & $56(5 / 9)$ & $44(4 / 9)$ \\
\hline Hipotonicidad & $12,5(7 / 57)$ & $71,4(5 / 7)$ & $28,6(2 / 7)$ \\
\hline Temperatura $>38^{\circ} \mathrm{C}$ & $7(4 / 57)$ & $25(1 / 4)$ & $75(3 / 4)$ \\
\hline Bradicardia fetal & $7(4 / 57)$ & $11,8(1 / 4)$ & $18,2(3 / 4)$ \\
\hline Cianosis central & $5,3(3 / 57)$ & $0(0 / 3)$ & $100(3 / 3)$ \\
\hline Síndrome emético & $3,5(2 / 57)$ & $50(1 / 2)$ & $50(1 / 2)$ \\
\hline Otros** & $7,01(4 / 57)$ & - & - \\
\hline \multicolumn{4}{|l|}{ Exámenes auxiliares* } \\
\hline Trombocitopenia & $20(11 / 55)$ & & - \\
\hline Hipoglicemia & $16,3(9 / 55)$ & & - \\
\hline Desaturación & $16,3(9 / 55)$ & & - \\
\hline Proteina $\mathrm{C}$ reactiva elevada ${ }^{\dagger}$ & $14,5(8 / 55)$ & & - \\
\hline Leucocitosis & $12,7(7 / 55)$ & & - \\
\hline Leucopenia & $9,2(5 / 55)$ & & - \\
\hline Anemia neonaal & $7,4(4 / 55)$ & & - \\
\hline I/T alterado & $3,6(2 / 55)$ & & - \\
\hline \multicolumn{4}{|c|}{ Procedimientos invasivos y situaciones que predispusieron su uso $\S$} \\
\hline Estancia en UCl & $50(12 / 24)$ & & - \\
\hline Cateterismo umbilical & $47,8(11 / 23)$ & & - \\
\hline Ventilación mecánica invasiva & $45,8(11 / 24)$ & & - \\
\hline Administración de antibióticos prolongadall & $41,7(10 / 24)$ & & - \\
\hline Tiempo de hospitalización prolongado" & $35,3(12 / 34)$ & & - \\
\hline Uso de surfactante & $26,5(9 / 34)$ & & - \\
\hline Reanimación neonatal & $29,4(10 / 34)$ & & - \\
\hline \multicolumn{4}{|l|}{ Comorbilidades* } \\
\hline Membrana hialina & $19,4(12 / 62)$ & & - \\
\hline Trastornos metabólicos & $19,4(12 / 62)$ & & - \\
\hline Hipoglicemia & $12,9(8 / 62)$ & & - \\
\hline Hipocalcemia & $3,2(2 / 62)$ & & - \\
\hline Hipoglicemia/hipocalcemia & $3,2(2 / 62)$ & & - \\
\hline Cardiopatías congénitas(PCA, CIA) & $14,5(9 / 62)$ & & - \\
\hline Hemorragia interventricular & $9,8(6 / 62)$ & & - \\
\hline Retinopatía del prematuro & $6,7(4 / 62)$ & & - \\
\hline Displasia broncopulmonar & $6,4(4 / 62)$ & & - \\
\hline Sufrimiento fetal agudo & $4,8(3 / 62)$ & & - \\
\hline Otros $\pi$ & $19(12 / 62)$ & & - \\
\hline \multicolumn{4}{|c|}{$\begin{array}{l}\text { Se evaluó más de una característica clínica, comorbilidad, procedimiento invasivo y examen auxiliar en un mismo neonato. } \\
\text { Regurgitación, signos de hipoperfusión tisular, taquicardia fetal y problemas de succión. } \\
\text { Elevado: } \geq 1,0 \mathrm{mg} / \mathrm{dL} \\
\text { Estice que mide la razón de inmaduros/neutrófilos, alterado }>0,16 \text {. } \\
\text { I Por encima del percentil evaluadas previo al diagnóstico de sepsis neonatal probable. } \\
\text { I Asfixia perinatal, policitemia, meningitis (no definida), incompatibilidad ABO, infecciones por TORCH (CMV), anormalidades del cordón umbilical } \\
\text { perforación intestinal, hernia inguinal, micosis pulmonar, hidrocefalea obstructiva, parálisis facial, hernia umbilical, criptorquidea. } \\
\text { CAP: Conducto arterial persistente CIA: Comunicación interauricular }\end{array}$} \\
\hline
\end{tabular}

de mayor complejidad médica según el nivel de cada establecimiento de salud.

Además de la elevada incidencia, destaca la elevada tasa de letalidad encontrada. Esta última aumenta en prematuros, en neonatos con extremadamente bajo peso al nacer y con peso por debajo del percentil 25. En
Estados Unidos del 2005 al 2008, la tasa de letalidad fue de $10,9 \%$, aumentando en pretérminos según (negros con $24,4 \%$ y blancos con $21,5 \%$ ) en comparación con neonatos a término (negro con $1,7 \%$ y blanco con $1,6 \%)^{(7)}$. En un estudio chileno, la mortalidad asociada a sepsis neonatal ha permanecido baja $(2,2 \%)^{(19)}$. La elevada incidencia de sepsis confirmada y la elevada mortalidad asociada 
en el HNAL denota una situación preocupante, siendo necesario instaurar medidas que disminuyan el impacto en los neonatos. Más aun considerando un patrón clínicolaboratorial no silente en la mayoría de neonatos con sepsis en el HNAL. Por otro lado, la tasa de mortalidad hallada fue similar a la reportada por otros países. En cuba, durante el 2003 al 2007, la tasa de mortalidad en los últimos 3 años ha tenido un aumento progresivo $(0,7$; 1,6 ; y 2 por cada 1000 nacidos vivos), representando la sepsis casi la mitad de los casos totales de las muertes ocurridas y la primera causa de mortalidad ${ }^{(20)}$.

Con relación al perfil microbiológico y terapéutico, los Staphylococcus spp. predominaron en los aislamientos de neonatos con sepsis precoz y tardía, evidenciando una elevada resistencia a oxacilina. El aislamiento de $S$. epidermidis y de $S$. Aureus también se ha reportado en estudios nacionales y en otros países ${ }^{(7,8)}$, y no solo son reportes aislados. La terapéutica administrada no estuvo acorde con el perfil microbiológico. A un $67 \%$ se inició amipicilina/amikacina o ampicilina/cefotaxima, resultando que a un $58 \%$ de neonatos se les rotó de antibióticos por mala evolución clínica/laboratorial o por resultado de hemocultivo. La prescripción empírica usada fue similar a lo recomendado por algunas guías americanas en la sepsis precoz ${ }^{(7)}$, no considerando que en Estados Unidos son frecuentes los GBS y Gram negativos ${ }^{(9,10)}$, y que es necesario el estudio del espectro bacteriano predominante en cada sede hospitalaria para definir el tratamiento empírico. La vancomicina fue usada con más frecuencia luego de la primera rotación de antibióticos, reduciendo la rotación a una nueva. Cuando se sospecha que hay SGN, algunas guías recomiendan que la vancomicina debe iniciarse empíricamente hasta que se confirme la susceptibilidad a oxacilina, debido a que los SGN presentan una alta tasa de resistencia a oxacilina ${ }^{(7,21)}$. Otros estudios sugieren iniciar el tratamiento empírico con cloxacilina, reservando la vancomicina para la sepsis causada por organismos con resistencia confirmada a oxacilina; siendo una medida importante para controlar la propagación de microorganismos resistentes a vancomicina ${ }^{(22)}$. También según el tipo de sepsis se puede considerar el tratamiento empírico. En general, en la sepsis precoz considerar cobertura para GBS y Gram negativos, y en la tardía para Staphylococcus spp., pero lo que definirá la elección del antibiótico es el patrón de resistencia local y los agentes infecciosos que se aíslan con más frecuencia. De este modo, el tratamiento antibiótico en la sepsis neonatal resulta un desafío debido a la resistencia bacteriana emergente ${ }^{(23,24)}$, el fracaso terapéutico ${ }^{(25)}$, el costo económico asociado (26), los efectos adversos ${ }^{(21,27)}$, y la elección del tratamiento empírico acorde a la vigilancia epidemiológica local.

Otro hallazgo importante es que las características descritas en sepsis confirmada son afines al perfil microbiológico reportado en el HNAL. Normalmente se espera encontrar presencia de Staphylococcus spp. en sepsis tardía, o secundario a procedimientos invasivos durante período prenatal (amniocentesis o la amnioinfusión) ${ }^{(28)}$ o durante hospitalización ${ }^{(7,29)}$. En el HNAL los aislamientos de Staphylococcus spp. predominaron tanto en sepsis precoz como tardía, un porcentaje considerable de estos neonatos estuvieron expuestos a procedimientos invasivos o a situaciones que predispusieron su uso (síndrome de distrés respiratorio, apnea, hospitalización prolongada, estancia en $\mathrm{UCl}$, entre otros). $S$. epidermidis desempeña un papel comensal en los humanos. Su presencia es importante en neonatos, mediante la inhibición de patógenos virulentos y la educación del sistema inmune innato. La interrupción de la barrera de la piel por medio de dispositivos médicos y la presión selectiva debido a los antibióticos, contribuyen a la conversión de $S$. epidermidis de un miembro de la microflora de la piel a un agente infeccioso. Sumado a la predisposición inmune que los neonatos tienen para las infecciones ${ }^{(28)}$. En este estudio, la exposición a procedimientos invasivos, tanto en la sepsis precoz y tardía, probablemente explique la alta frecuencia de aislamientos de SGN. Aparte de fortalecer las medidas higiénicas y la vigilancia epidemiológica para evitar la propagación de gérmenes intrahospitalarios, la reducción del uso indiscriminado de procedimientos invasivos podría ser beneficiosa.

En este estudio no es posible establecer si el tratamiento empírico instaurado ha contribuido con la elevada tasa de letalidad evidenciada. Otra limitación es que se utilizó una fuente secundaria, predisponiendo a que la información consignada en las historias clínicas tenga sesgos. Además, en este estudio para definir sepsis confirmada solo fue necesario un hemocultivo positivo, existiendo la posibilidad de que sean aislamientos contaminados. Sin embargo, todos los casos de sepsis confirmada presentaron previamente características clínicas, alteraciones de exámenes auxiliares y factores neonatales-maternos compatibles con sepsis probable. Esto reduce en gran magnitud la posibilidad que los casos sean contaminación.

En conclusión, se encontró una elevada incidencia de sepsis neonatal confirmada, con una elevada tasa de letalidad. La terapéutica aplicada no estuvo acorde al perfil microbiológico. Se recomienda realizar una vigilancia microbiológica activa y permanente, no realizar procedimientos invasivos indiscriminadamente, establecer el tratamiento empírico acorde al patrón de resistencia bacteriana de cada hospital, y evaluar su efectividad. Todo ello con el fin de disminuir la morbimortalidad de la sepsis neonatal en el HNAL, y a la vez contribuir con la disminución de la mortalidad neonatal e infantil en nuestro país. 
Contribuciones de autoría: AGAG contribuyó con la concepción y diseño del artículo, recolección de resultados, análisis de resultados, redacción, aprobación de su versión final y asesoría estadística. KMAM contribuyó con la concepción y diseño del artículo, análisis de resultados, redacción, aprobación de su versión final, revisión crítica del artículo. DMAA contribuyó con la recolección de resultados, análisis de resultados, redacción, aprobación de su versión final, revisión crítica del artículo. VBC contribuyó con la concepción y diseño del artículo, análisis de resultados, redacción, aprobación de su versión final, revisión crítica del artículo, aporte de pacientes o material de estudio, asesoría técnica o administrativa.

Fuente de financiamiento: autofinanciado.

Declaración de conflictos de interés: los autores declaran no tener conflictos de interés.

\section{REFERENCIAS BIBLIOGRÁFICAS}

1. Velásquez Hurtado JE, Kusunoki Fuero L, Paredes Quiliche TG, Hurtado La Rosa R, Rosas Aguirre AM, Vigo Valdez WE. Mortalidad neonatal, análisis de registros de vigilancia e historias clínicas neonatales del año 2011 en Huánuco y Ucayali, Perú. Rev Peru Med Exp Salud Publica. 2014; 31(2): 228-36.

2. Instituto Nacional de Estadística e Informática. Perú: Encuesta Demográfica y de Salud FamiliarENDES 2013. Lima: INEI; 2014. Disponible en: https://dhsprogram. com/pubs/pdf/FR299/FR299.pdf

3. Avila J. Vigilancia epidemiológica perinatal y neonatal, Perú, año 2014 (a la SE 13). Bol Epidemiol (Lima). 2014;23(13):247-49.

4. Avila J. Vigilancia epidemiológica perinatal y neonatal, Perú 2013. Bol Epidemiol (Lima). 2013;22(52):107983.

5. Ministerio de Salud, Dirección General de Epidemiología. Subsistema nacional de vigilancia epidemiológica perinatal y neonatal. Lima: MINSA/DGE; 2009.

6. Obiero CW, Seale AC, Berkley JA. Empiric treatment of neonatal sepsis in developing countries. Pediatr Infect Dis J. 2015;34(6):659-61. doi:10.1097/ INF.0000000000000692.

7. Simonsen KA, Anderson-Berry AL, Delair SF, Davies HD. Early-Onset Neonatal Sepsis. Clin Microbiol Rev. 2014; 27(1):21-47. doi: 10.1128/ CMR.00031-13.

8. Shimabuku R, Velásquez $P$, Yábar J, Zerpa R, Arribasplata G, Fernández $S$, et al. Etiología y susceptibilidad antimicrobiana de las infecciones neonatales. An Fac med. 2004;65(1):19-24.

9. Weston EJ, Pondo T, Lewis MM, Martell-Cleary P, Morin C, Jewell $\mathrm{B}$, et al. The burden of invasive earlyonset neonatal sepsis in the United States, 2005-2008. Pediatr Infect Dis
J. 2011; 30(11):937-41. doi: 10.1097/ INF.0b013e318223bad2.

10. Bizzarro MJ, Raskind C, Baltimore RS, Gallagher PG. Seventy-five years of neonatal sepsis at Yale: 1928-2003. Pediatrics.2005;116(3):595-602.

11. Saltigeral SP, Valenzuela FAMC, Avendaño E, Plascencia S, Martínez D. Agentes causales de sepsis neonatal temprana y tardía: una revisión de diez años en el "Hospital Infantil Privado". Rev Enferm Infecc Pediatr. 2007; 20(80):99-105.

12. Pérez Y, Clemades AM, Mederos Y, Navarro M, Arbelo I, Molina O. Sepsis neonatal grave en una unidad de cuidados intensivos. Rev Cubana Pediatr. 2015;87(1):50-60.

13. Ticona M, Huanco D. Mortalidad perinatal hospitalaria en el Perú: Factores de riesgo. Rev Chil Obstet Ginecol. 2005;70(5):313-17.

14. Ticona-Rendón M, Huanco-Apaza D. Factores de riesgo de la mortalidad perinatal en hospitales del Ministerio de Salud del Perú. Rev Cubana Obstet Ginecol. 2011;37(3): 431-43.

15. Cohen-Wolkowiez M, Moran C, Benjamin DK, Cotten CM, Clark RH, Benjamin DK Jr, et al. Early and late onset sepsis in late preterm infants. Pediatr Infect Dis J. 2009;28(12):1052-6.

16. Stoll BJ, Hansen NI, Sanchez PJ, Faix RG, Poindexter BB, Van Meurs KP, et al. Early onset neonatal sepsis: the burden of group B streptococcal and $E$. coli disease continues. Pediatrics. 2011;127(5):817-26. 10.1542/ peds.2010-2217.

17. Perú, Ministerio de Salud. Guía práctica clínica para la atención del recién nacido con sepsis [internet]. Lima: MINSA; 2013 [citado el 15 de abril de 2015]. Disponible en: http://www.hospitalcayetano. gob.pe/transparencia/images/ stories/resoluciones/RD/RD2013/ rd_451_2013.pdf
18. Vergnano S, Sharland M, Kazembe P, Mwansambo C, Heath PT. Neonatal sepsis: an international perspective. Arch Dis Child Fetal Neonatal Ed. 2005;90(3):F220-4

19. Tapia I JL, Reichhard T C, Saldías R MI, Abarzúa C F, Pérez A ME, González M A, et al. Sepsis neonatal en la era de profilaxis antimicrobiana prenatal. Rev Chil Infectol. 2007;24(2):111-6.

20. Fernandez N, Duque de estrada J, Diaz F. Morbilidad y mortalidad por sepsis neonatal precoz. Rev Cubana Pediatr. 2010;82(2):0-0.

21. Jacqz-Aigrain E, Zhao W, Sharland $M$, van den Anker JN. Use of antibacterial agents in the neonate: 50 years of experience with vancomycin administration. Semin Fetal Neonatal Med. 2013;18(1):28-34. doi: $10.1016 /$ j.siny.2012.10.003.

22. Lawrence SL, Roth V, Slinger $R$, Toye B, Gaboury I, Lemyre B. Cloxacillin versus vancomycin for presumed late-onset sepsis in the Neonatal Intensive Care Unit and the impact upon outcome of coagulase negative staphylococcal bacteremia: a retrospective cohort study. BMC Pediatr. 2005; 5:49. doi: 10.1186/1471-2431-5-49.

23. Tzialla C, Borghesi A, Pozzi M, Stronati M. Neonatal infections due to multi-resistant strains: Epidemiology, current treatment, emerging therapeutic approaches and prevention. Clin Chim Acta. 2015;451(Pt A):71-7. doi: 10.1016/j. cca.2015.02.038.

24. Alvarado-Gamarra AG, AlcaláMarcos KM, Alvarado-Gamarra PK, Champi-Merino R. Riesgo de aparición de cepas de Staphylococcus aureus resistente a vancomicina en pacientes hospitalarios de un hospital del Perú, 2008. CIMEL. 2010;15(2):59-62. 
25. Reyna J, Ortiz F. Therapeutic failure of the ampicillin plus aminoglycoside scheme in the treatment of early neonatal sepsis. Arch Med Res. 2008; 39(5):546-7. doi: 10.1016/j. arcmed.2008.02.009.

26. Reyna-Figueroa J, Ortiz-Ibarra FJ, Estéves Jaramillo A, Reyna-Figueroa J. Costo económico marginal del fracaso terapéutico con ampicilina más amikacina en el tratamiento de la sepsis neonatal temprana. An Pediatr
(Barc). 2009;71(1):54-9. 10.1016/j. anpedi.2009.03.013.

27. Adelman RD, Wirth F, Rubio T. A controlled study of the nephrotoxicity of mezlocillin and gentamicin plus ampicillin in the neonate. $\mathrm{J}$ Pediatr. 1987; $111(6$ Pt 1):888-93.

28. André P, Thébaud B, Guibert $M$, Audibert F, Lacaze-Masmonteil T, Dehan M. Maternal-fetal staphylococcal infections: a series report. Am J Perinatol. 2000;17(8):423-7.
29. Dong Y, Speer CP. The role of Staphylococcus epidermidis in neonatal sepsis: guarding angel or pathogenic devil? Int $\mathrm{J}$ Med Microbiol. 2014;304(5-6):513-20. doi: 10.1016/j.ijmm.2014.04.013.

Correspondencia: Giancarlo Alvarado Gamarra

Dirección: Jr. Salaverry 380, Dpto. 903, Magdalena del Mar. Lima, Perú. Teléfono: (511) 993376826

Correo electrónico: galvaradogamarra@ gmail.com

\section{http://facebook.com/rpmesp}

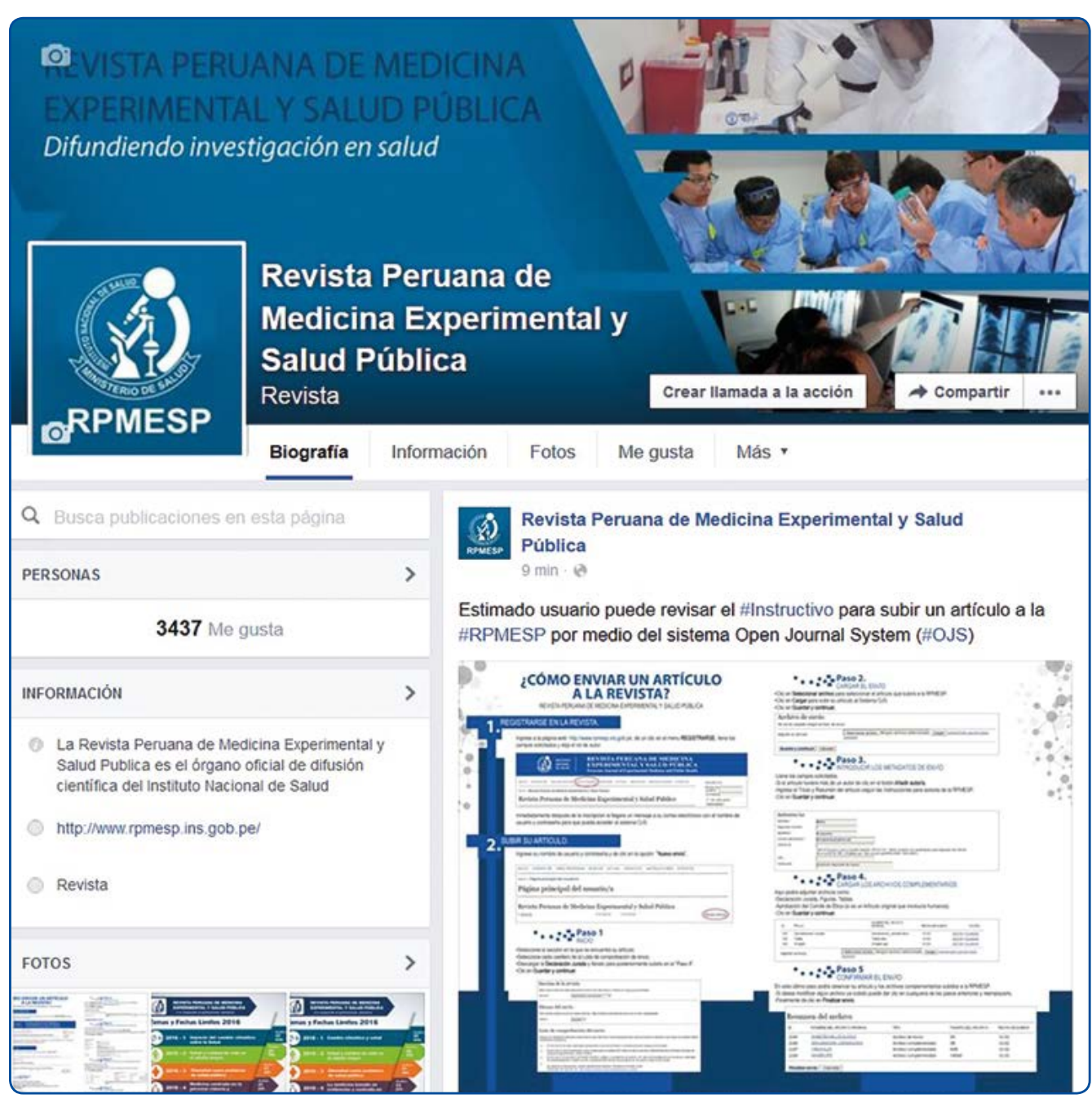

